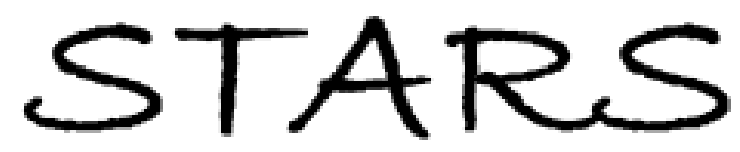

University of Central Florida

STARS

$1-1-2013$

\title{
Near-infrared femtosecond laser machining initiated by ultraviolet multiphoton ionization
}

X. Yu

Q. Bian

B. Zhao

Z. Chang

University of Central Florida

P. B. Corkum

See next page for additional authors

Find similar works at: https://stars.library.ucf.edu/facultybib2010

University of Central Florida Libraries http://library.ucf.edu

This Article is brought to you for free and open access by the Faculty Bibliography at STARS. It has been accepted for inclusion in Faculty Bibliography 2010 s by an authorized administrator of STARS. For more information, please contact STARS@ucf.edu.

\section{Recommended Citation}

Yu, X.; Bian, Q.; Zhao, B.; Chang, Z.; Corkum, P. B.; and Lei, S., "Near-infrared femtosecond laser machining initiated by ultraviolet multiphoton ionization" (2013). Faculty Bibliography 2010 s. 4906.

https://stars.library.ucf.edu/facultybib2010/4906

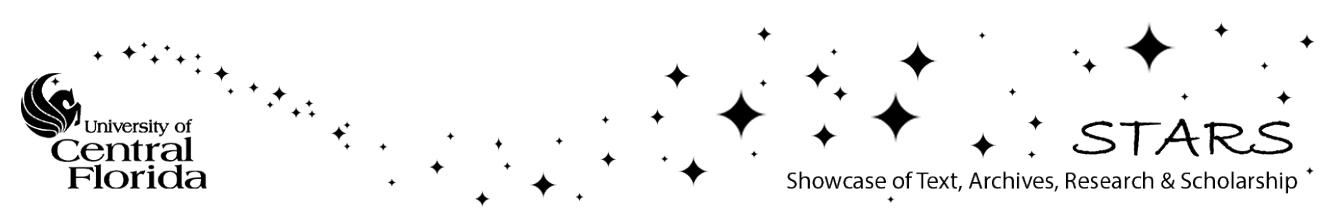


Authors

X. Yu, Q. Bian, B. Zhao, Z. Chang, P. B. Corkum, and S. Lei 


\section{Near-infrared femtosecond laser machining initiated by ultraviolet multiphoton ionization}

Cite as: Appl. Phys. Lett. 102, 101111 (2013); https://doi.org/10.1063/1.4794946

Submitted: 16 October 2012 . Accepted: 26 February 2013 . Published Online: 14 March 2013

X. Yu, Q. Bian, B. Zhao, Z. Chang, P. B. Corkum, and S. Lei

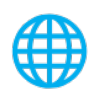

\section{ARTICLES YOU MAY BE INTERESTED IN}

Femtosecond laser three-dimensional micro- and nanofabrication

Applied Physics Reviews 1, 041303 (2014); https://doi.org/10.1063/1.4904320

Ablation of solids by femtosecond lasers: Ablation mechanism and ablation thresholds for metals and dielectrics

Physics of Plasmas 9, 949 (2002); https://doi.org/10.1063/1.1447555

Laser-induced breakdown by impact ionization in $\mathrm{SiO}_{2}$ with pulse widths from 7 ns to 150 fs Applied Physics Letters 64, 3071 (1994); https://doi.org/10.1063/1.111350

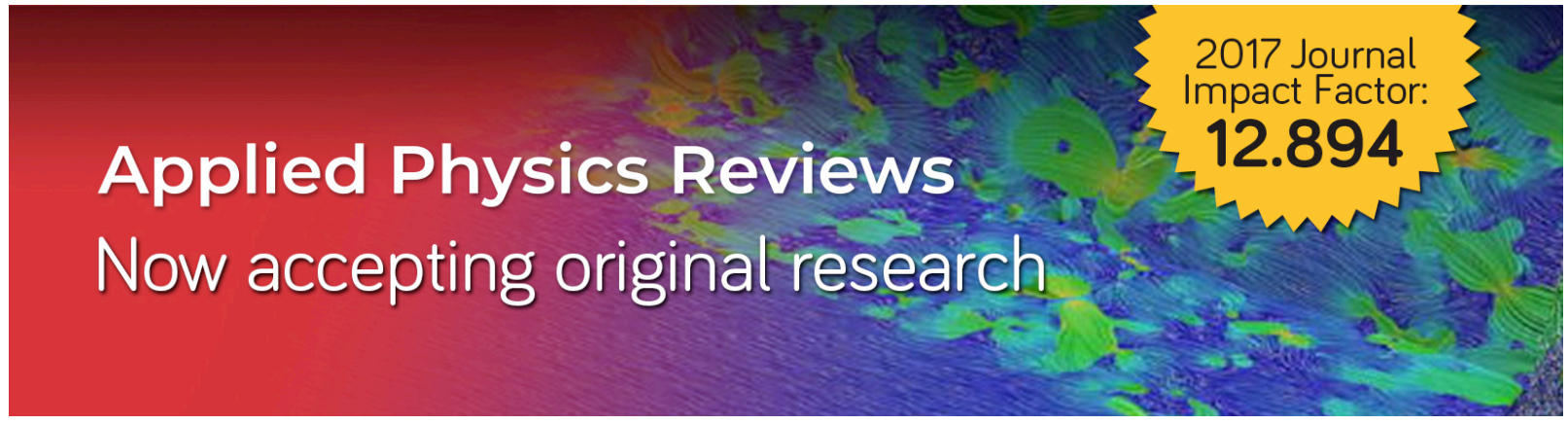




\title{
Near-infrared femtosecond laser machining initiated by ultraviolet multiphoton ionization
}

\author{
X. Yu, ${ }^{1}$ Q. Bian, ${ }^{1}$ B. Zhao,${ }^{2}$ Z. Chang,${ }^{3}$ P. B. Corkum,,${ }^{4,5}$ and S. Lei ${ }^{1}$ \\ ${ }^{1}$ Industrial and Manufacturing Systems Engineering, Kansas State University, Manhattan, Kansas 66506, USA \\ ${ }^{2}$ Department of Physics and Astronomy, University of Nebraska Lincoln, Lincoln, Nebraska 68588, USA \\ ${ }^{3}$ CREOL and Department of Physics, University of Central Florida, Orlando, Florida 32816, USA \\ ${ }^{4}$ National Research Council of Canada, Ottawa, Ontario KlA OR6, Canada \\ ${ }^{5}$ University of Ottawa, Ottawa, Ontario KIN 6N5, Canada
}

(Received 16 October 2012; accepted 26 February 2013; published online 14 March 2013)

\begin{abstract}
We report on the experimental study of microstructures fabricated on the surface of fused silica by two femtosecond laser pulses, a tightly focused $266 \mathrm{~nm}$ beam followed by a loosely focused $800 \mathrm{~nm}$ beam. By setting the fluence of each pulse below the damage threshold, visible microstructures are fabricated using the combined beams. Our results suggest that the ultraviolet pulse generates seed electrons through multiphoton absorption, and the near-infrared pulse utilizes these electrons to cause damage by avalanche ionization. (C) 2013 American Institute of Physics. [http://dx.doi.org/10.1063/1.4794946]
\end{abstract}

Femtosecond (fs) laser micromachining has been widely studied for various applications because of its unique advantages such as reduced thermal effect. ${ }^{1-3}$ Due to the high nonlinearity of the interaction between femtosecond laser and transparent materials, e.g., fused silica, damage features are localized within focal volumes, ${ }^{4,5}$ and nanoscale features can be achieved by pulse energy control, ${ }^{6}$ use of near-field scanning microscopes, ${ }^{7}$ self-focusing, ${ }^{8}$ etc. Fundamentally, the minimum feature size is determined by the diffraction limit on the order of $\lambda / \mathrm{NA}$, where $\lambda$ is laser wavelength and NA is the numerical aperture of focusing optics. Using high NA objectives, small holes with diameters of $200 \mathrm{~nm}$ are fabricated. ${ }^{9}$ Alternatively, using extreme ultraviolet (XUV) pulses of tens of nanometers wavelength generated through high harmonic generation (HHG) ${ }^{10,11}$ feature size can be controlled at the nanometer level. However, due to the low conversion efficiency of HHG, direct machining of dielectrics using HHG is a challenge. Furthermore, for mass production purposes, separation of laser beams to form several foci can increase production speed, so a machining technique requiring lower pulse energy, even below damage threshold, is desired.

Since laser pulses with a shorter wavelength have a larger cross section to excite free electrons from the valance band to the conduction band by photoionization, ${ }^{11}$ it is possible to use a short wavelength pulse to generate seed electrons and a long wavelength pulse to cause an electron avalanche. ${ }^{12}$ With the help of the long wavelength pulse, the energy of the short wavelength pulse might be kept below its damage threshold, and damage can only form within the seeding volume defined by the short wavelength pulse. This provides a possible route to XUV (or even shorter wavelength) ${ }^{13,14}$ laser nanomachining with energy below damage threshold.

Several studies have been carried out to investigate the effects of combining two femtosecond pulses or two-color nanosecond pulses on machining of different materials, and their main concern was ablation enhancement and threshold reduction. ${ }^{15-19}$ This paper focuses on the multiphoton and avalanche processes by an ultraviolet-near-infrared
(UV-NIR) pulse train to reveal the mechanism of damage formation and the control of damage size defined by the short wavelength pulse. We choose UV light as seeding pulses instead of XUV because no vacuum devices are needed. The results discussed in this paper are universal and can be applied to an XUV-NIR setup for nanoscale machining.

Fig. 1 shows the experimental setup. A fs laser system delivers $60 \mathrm{fs}$ (full width at half maximum, FWHM) laser pulses at the center wavelength of $800 \mathrm{~nm}$ with the maximum pulse energy of $4.3 \mathrm{~mJ}$ and repetition rate of $1 \mathrm{kHz}$. The beam is split into two arms (UV and NIR arm) after a beam splitter. $90 \%$ of the beam is reflected (UV arm) and directed through a third harmonic generation (THG) module (Eksma Optics). The barium borate (BBO) crystal generates the second harmonic $(400 \mathrm{~nm})$ of the fundamental (NIR) beam. A

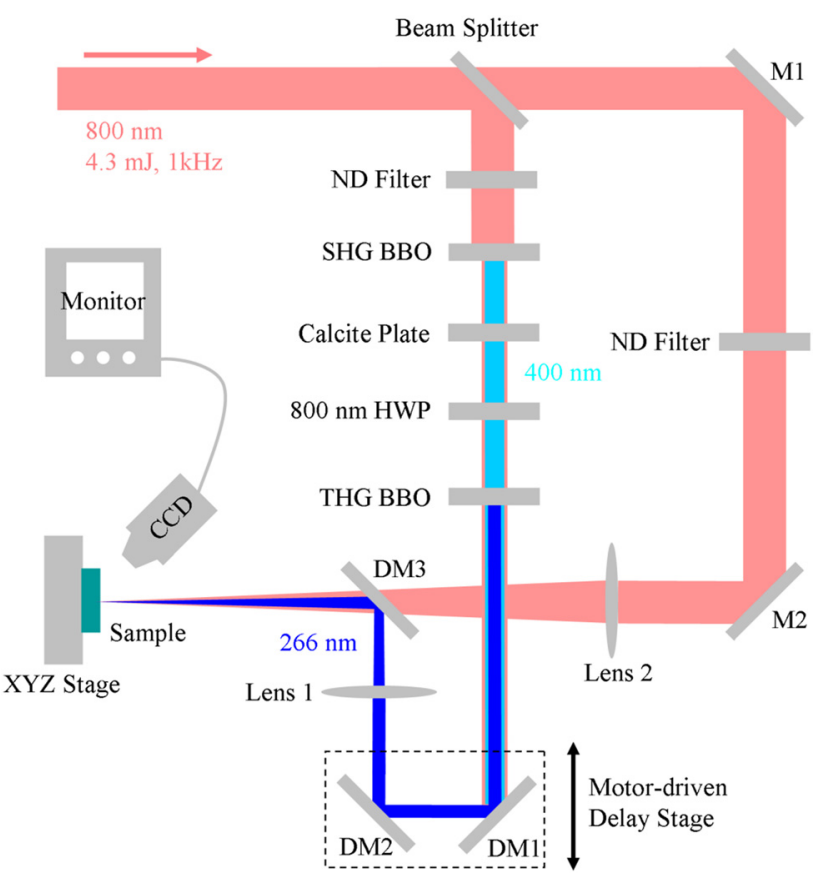

FIG. 1. Experimental setup. 
calcite plate is used to compensate for the group velocity delay between the second harmonic and the NIR pulses. A zero-order wave-plate (half-wave at $800 \mathrm{~nm}$ and full-wave at $400 \mathrm{~nm}$ ) makes the 400 and the $800 \mathrm{~nm}$ polarize in the same direction so that the $266 \mathrm{~nm}$ (UV) pulses is generated in another BBO crystal through sum frequency generation. The pulse duration of the UV pulses is estimated to be $70 \mathrm{fs}$. The maximum UV pulse energy is $20 \mu \mathrm{J}$, resulting in a conversion efficiency of $0.5 \%$. The energy is adjusted by a neutral-density (ND) filter before the first BBO. After the THG module, only the UV beam is efficiently reflected by two dichroic mirrors (DM1 and DM2, Layertec) mounted on a motor-driven delay stage. Then the UV beam is focused by a lens $(50 \mathrm{~mm}$ focal length). The $800 \mathrm{~nm}$ beam in the NIR arm is focused by another lens ( $200 \mathrm{~mm}$ focal length), and its energy is controlled by an ND filter.

The focused UV and NIR beams are combined with another dichroic mirror (DM3) and both foci are carefully aligned on the sample surface. The focal spot size $\left(2 w_{0}\right)$ for the UV and NIR measured by the knife-edge method is $9.2 \mu \mathrm{m}$ and $25.4 \mu \mathrm{m}$, respectively, where $w_{0}$ is the $1 / \mathrm{e}^{2}$ radius. The samples are square fused silica plates of $25.4 \mathrm{~mm}$ wide and $2 \mathrm{~mm}$ thick (Corning 7980). The samples are mounted on a motor-driven three-axis stage. To temporally overlap the UV and NIR beams, a $200 \mu \mathrm{m}$ thick BBO crystal is inserted after DM3 and the difference frequency generation of $400 \mathrm{~nm}$ from the UV and NIR is observed. Zero delay is defined when the generated $400 \mathrm{~nm}$ light is the strongest, with an accuracy of $\sim 30 \mathrm{fs}$.

In the experiments, both the UV and NIR energy fluence are kept below their respective damage threshold $\left(0.87 \mathrm{~J} / \mathrm{cm}^{2}\right.$ for UV and $2.17 \mathrm{~J} / \mathrm{cm}^{2}$ for NIR), so that no visible damage is observed with a charge-coupled device (CCD) camera when only one beam is applied. At a fixed NIR fluence and a relative delay to the UV pulse, UV energy is carefully adjusted (still kept below its damage threshold) to a level that visible damages are starting to appear. By this method, the damage threshold energy range can be narrowed down to an acceptable level. It should be noted that different methods have been used in determining damage threshold, such as direct observation, plasma emission, and light transmission..$^{12,17,20}$ Our method is similar to the one used in Ref. 20.

Fig. 2 shows the dependence of the UV damage threshold on time delay between the UV and NIR pulses for different NIR fluences. Positive values in delay mean UV pulses are irradiated on sample surfaces before NIR pulses. At the highest NIR fluence, the UV damage threshold reaches a minimum of $0.25 \mathrm{~J} / \mathrm{cm}^{2}$ at $60 \mathrm{fs}$ delay, which is $71 \%$ lower than that in the normal case (damage threshold by UV alone). Actually, it is found that if NIR fluence is kept $2 \%$ below its damage threshold, the lowest fluence needed at 60 fs delay for UV is $88 \%$ lower than that in the normal case. Away from this "optimal" delay, UV damage threshold increases, however, in an asymmetric manner: It returns to the normal value faster from the negative delay side than from the positive delay side. With NIR fluence decreasing, more UV energy is required to damage the sample, i.e., the "effective" damage threshold increases.

In ultrafast laser ablation of dielectric materials, it is well known that multiphoton and avalanche ionization play a key role. In multiphoton ionization, UV pulses are more efficient

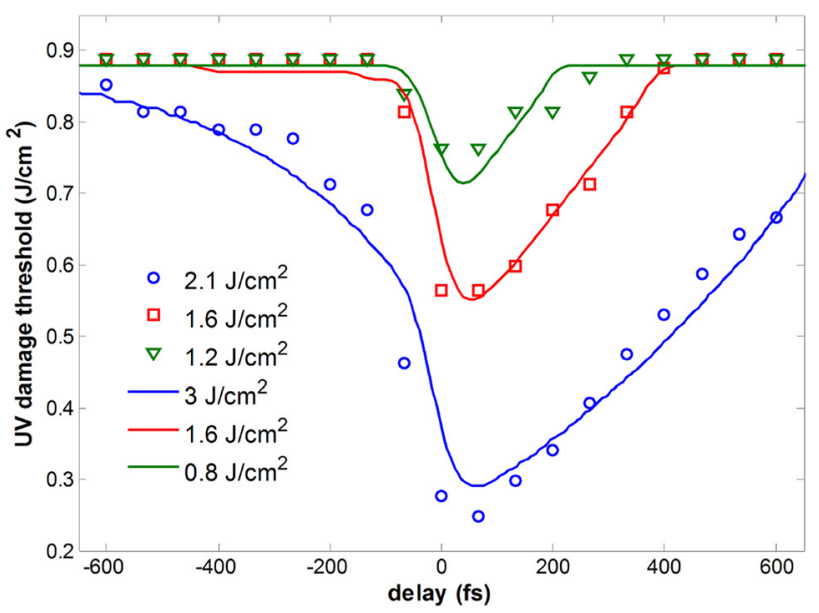

FIG. 2. Dependence of UV damage threshold on the time delay at different NIR fluences. Dots: experimental data. Curves: simulation data. $\alpha_{U V}=2$ $\mathrm{cm}^{2} / \mathrm{J}, \quad \alpha_{N I R}=0.9 \quad \mathrm{~cm}^{2} / \mathrm{J}, \quad t_{p, U V}=70 \quad$ fs, $\quad t_{p, N I R}=60 \quad$ fs, $\quad \tau=250 \quad \mathrm{fs}$, $n_{\text {critical }}=1.7 \times 10^{21} / \mathrm{cm}^{3}$.

in generating free electrons than NIR pulses. Specifically, for fused silica (with a bandgap of $9 \mathrm{eV}$ ), only 2 photons are needed for $266 \mathrm{~nm}$ (UV) light to generate an electron from valance band to conduction band, while for $800 \mathrm{~nm}$ (NIR) light, this number is 6 , resulting a much higher cross section for UV. After this ionization process, these free electrons will be self-trapped to form exitons (STEs) in $\sim 150 \mathrm{fs}$, which have a life time of 30-300 ps. ${ }^{12,17}$ It is suggested that these STEs generated by a pre-pulse can be again excited to become free electrons. ${ }^{12}$ Damage forms when free electron density reaches the critical density value $\left(\sim 10^{21} / \mathrm{cm}^{3}\right)$.

In Fig. 2, for every NIR fluence, the UV threshold reaches a minimum value at $\sim 60 \mathrm{fs}$ and increases faster in the negative delay direction than in the positive delay direction. The number of free electron density reaches the maximum at the end of the $60 \mathrm{fs} \mathrm{UV}$ pulse because the lifetime of the electrons is much longer than the laser pulse duration, therefore the minimum UV fluence is required at the $60 \mathrm{fs}$ delay. Because UV is more sufficient in generating seed electrons, the asymmetric shape is seen at each NIR fluence. It is noted that UV damage threshold is higher at zero delay. This is probably because the free electrons need some time to build up, and the maximum number is reached after the UV pulse. Another feature seen from this figure is the dependence of UV threshold on NIR fluence. A higher NIR fluence provides more avalanche ionization and can re-excite more free electrons from STEs, ${ }^{12}$ and therefore, a lower UV threshold is observed. It is found that with a high NIR fluence $\left(2.1 \mathrm{~J} / \mathrm{cm}^{2}\right)$, the UV damage threshold returns to its normal value at $\sim 27.3 \mathrm{ps}$, which is similar to the lifetime of STEs in accordance with Ref. 12.

A simple rate equation describing the evolution of free electron density is used to testify our assumption discussed above

$$
\frac{d n}{d t}=W_{P I}\left(I_{U V}\right)+\alpha_{U V} I_{U V} n+W_{P I}\left(I_{N I R}\right)+\alpha_{N I R} I_{N I R} n-\frac{n}{\tau},
$$

where $n$ is the free electron density, $W_{P I}$ is the multiphoton ionization rate calculated from the Keldysh theory, ${ }^{11} \alpha_{U V}$ 
and $\alpha_{N I R}$ are the avalanche ionization rates for UV and NIR pulses, $\tau$ is the electron recombination time, and $I_{U V}$ and $I_{N I R}$ are the intensity for UV and NIR pulses

$$
I=I_{0} \exp \left(-4 \ln 2 \frac{\left(t-t_{d}\right)^{2}}{t_{p}^{2}}\right)
$$

where $I_{0}$ is the peak intensity, $t_{d}$ is the delay between UV and NIR pulses, and $t_{p}$ is the pulse duration (FWHM). $I_{0}$ can be calculated from fluence and pulse duration, i.e., $I_{0}=F / t_{p}$. This model has been widely used to investigate the interaction between femtosecond lasers and dielectrics. ${ }^{17,20,21}$ The damage threshold is determined when the free electron density reaches the critical density $\left(1.7 \times 10^{21} / \mathrm{cm}^{3}\right){ }^{20,22}$ The simulation results are shown in Fig. 2, which fit well to the experimental data. The difference of NIR fluence between experimental data and simulation is probably due to the Gaussian shape of the beam, which is not considered in the simulation. It should be noted that in this model, only multiphoton and avalanche ionization are considered, and the ionization term is found to be essential for a good fitting. This indicates that avalanche ionization plays a key role.

Since damage forms as a combined effect of UV and NIR pulses, the damage size is determined by the size of the area where the two beams overlap. In Fig. 3(a), the NIR spot size is deliberately enlarged by moving its focus forward by $8.2 \mathrm{~mm}$, resulting in a $330 \mu \mathrm{m}$ diameter spot on the fused silica surface. The UV focus is fixed on the surface and in the central portion of the NIR spot. The sample moves towards left at a constant speed of $0.5 \mathrm{~mm} / \mathrm{s}$, and while moving, DM3 is tilted vertically back and forth while the NIR spot is kept fixed, and thus a wavy structure is written on the sample surface. On the contrary, in Fig. 3(b), while keeping the UV spot fixed and tilting M2, the NIR spot is shifting vertically, and in contrast, a straight line is written. The temporal delay difference by tilting those mirrors is calculated to be less than $1 \mathrm{fs}$. Since the seeding beam such as the UV or XUV can be focused to a spot much smaller than that of NIR, this paves a way for machining features on scales that cannot be achieved with NIR beam alone.

Fig. 4 shows the relationship between the average linewidth and UV fluence at different delays and NIR fluences.

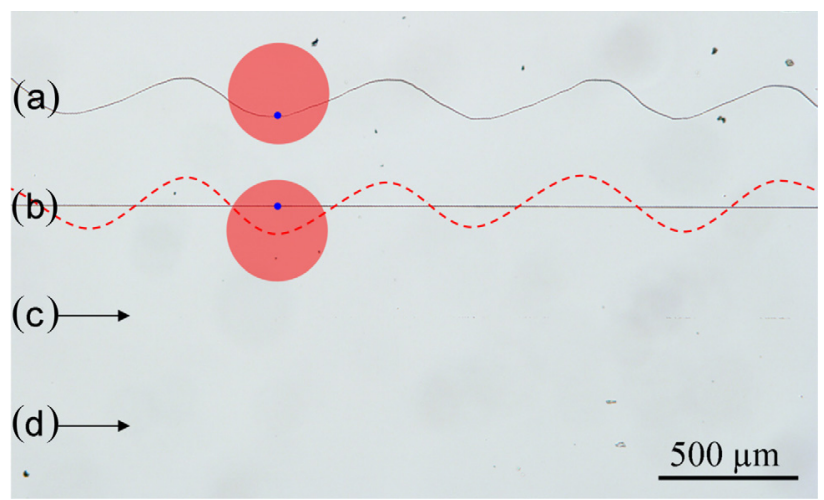

FIG. 3. Optical images of different structures fabricated by a combination of UV and NIR pulses. (a) Moving UV spot while keeping NIR spot fixed. (b) Moving NIR spot while keeping UV spot fixed. No structure can be seen using either (c) UV or (d) NIR beam individually to write straight lines.

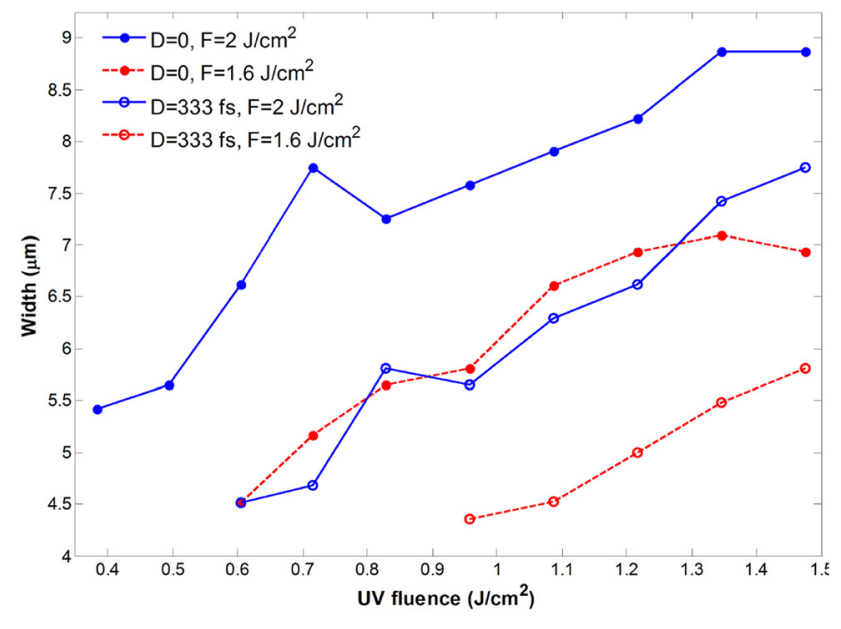

FIG. 4. Relationship between linewidth and UV fluence at different delays (D) and NIR fluences (F).

It can be seen that at each NIR fluence, the linewidth increases as UV fluence increases. This is due to the Gaussian shape of the focal spot. At each UV fluence, the linewidth is larger at zero delay and higher NIR fluence and is smaller at $333 \mathrm{fs}$ delay and lower NIR fluence. It is also noted that similar linewidth may be achieved with either zero delay and low NIR fluence or 333 fs delay and high NIR fluence. The narrowest, continuous linewidth we can achieve from a single UV beam is $4.7 \mu \mathrm{m}$, which is similar to the narrowest linewidth with the two-pulse configuration.

The fact that similar linewidth can be achieved by controlling the multiphoton and avalanche ionization is manifested in Fig. 5. Linewidth close to that by UV only (Fig. $5(\mathrm{a})$ ) is demonstrated under four sets of different conditions when UV and NIR pulses are combined. Another interesting feature is that with the combination of UV and NIR pulses at different delays, line quality differs. Fig. 5(a) shows a line written by a single UV beam with an average width of $4.7 \mu \mathrm{m}$. Due to the motion error of the XYZ stage and fluctuation of laser power, rough edges and small vertical grooves with an interval of $2 \mu \mathrm{m}$ can be seen. These grooves are caused by successive pulses with the repetition rate of $1 \mathrm{kHz}$
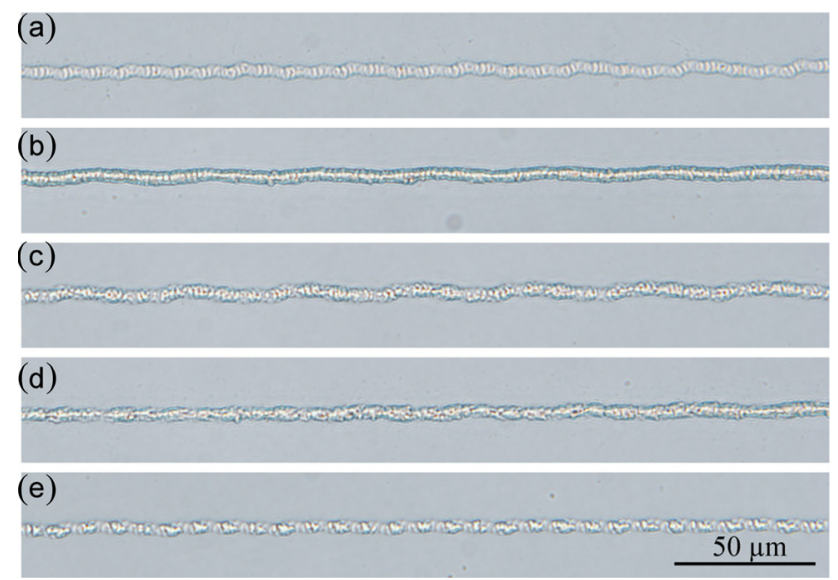

FIG. 5. Optical images of lines with similar widths written by (a) UV beam only, (b) UV with $2 \mathrm{~J} / \mathrm{cm}^{2} \mathrm{NIR}$ at zero delay, (c) UV with $1.6 \mathrm{~J} / \mathrm{cm}^{2} \mathrm{NIR}$ at zero delay, (d) UV with $2 \mathrm{~J} / \mathrm{cm}^{2} \mathrm{NIR}$ at 333 fs delay, and (e) UV with $1.6 \mathrm{~J} / \mathrm{cm}^{2}$ NIR at 333 fs delay. UV fluence: (a) $1.6 \mathrm{~J} / \mathrm{cm}^{2}$, (b) $0.33 \mathrm{~J} / \mathrm{cm}^{2}$, (c) and (d)) $0.61 \mathrm{~J} / \mathrm{cm}^{2}$, and (e) $1.2 \mathrm{~J} / \mathrm{cm}^{2}$. 
on a moving sample with the speed of $2 \mathrm{~mm} / \mathrm{s}$. With the combination of UV and high fluence NIR pulses at zero delay (Fig. 5(b)), the edges become smoother and those vertical grooves disappear. The mechanism of these smooth lines is unknown. One possible reason is the re-modification by the NIR beam, especially when the NIR fluence is high.

In summary, we have studied experimentally the micromachining of fused silica by a combination of UV and NIR pulses. With the help of NIR pulses at the fluence close to its damage threshold, the damage threshold of UV pulses can be lowered by $88 \%$ at $~ 60$ fs delay. Moving away from this optimal delay, UV damage threshold approaches its single beam threshold. It is demonstrated that the damage caused by this two-beam configuration has similar feature size as that produced by a single UV beam, and the line quality can be controlled by the degree of seed injection by UV multiphoton absorption and the subsequent NIR avalanche ionization. Using this approach, nanoscale machining is possible by replacing the UV beam with XUV beams generated by HHG with the fluence well below their single beam damage threshold.

This work was funded by the Army Research Office.

${ }^{1}$ B. N. Chichkov, C. Momma, S. Nolte, F. von Alvensleben, and A. Tünnermann, Appl. Phys. A 63, 109 (1996).

${ }^{2}$ R. R. Gattass and E. Mazur, Nature Photon. 2, 219 (2008).

${ }^{3}$ F. He, Y. Cheng, Z. Z. Xu, Y. Liao, J. Xu, H. Y. Sun, C. Wang, Z. H. Zhou, K. Sugioka, K. Midorikawa, Y. H. Xu, and X. F. Chen, Opt. Lett. 35, 282 (2010).
${ }^{4}$ C. B. Schaffer, A. Brodeur, J. F. Garcia, and E. Mazur, Opt. Lett. 26, 93 (2001).

${ }^{5}$ H. Varel, D. Ashkenasi, A. Rosenfeld, M. Wahmer, and E. E. B. Campbell, Appl. Phys. A 65, 367 (1997).

${ }^{6}$ A. Joglekar, H. Liu, E. Meyhofer, G. Mourou, and A. Hunt, Proc. Natl. Acad. Sci. U.S.A. 101, 5856 (2004).

${ }^{7}$ S. Nolte, B. N. Chichkov, H. Welling, Y. Shani, K. Lieberman, and H. Terkel, Opt. Lett. 24, 914 (1999).

${ }^{8}$ S. I. Kudryashov, G. Mourou, A. Joglekar, J. F. Herbstman, and A. J. Hunt, Appl. Phys. Lett. 91, 141111 (2007).

${ }^{9}$ Y. V. White, X. Li, Z. Sikorski, L. M. Davis, and W. Hofmeister, Opt. Express 16, 14411 (2008).

${ }^{10}$ P. B. Corkum, Phys. Rev. Lett. 71, 1994 (1993).

${ }^{11}$ L. V. Keldysh, Sov. Phys. JETP 20, 1307 (1965).

${ }^{12}$ D. Grojo, M. Gertsvolf, S. Lei, T. Barillot, D. M. Rayner, and P. B. Corkum, Phys. Rev. B 81, 212301 (2010).

${ }^{13}$ T. Mocek, J. Polan, P. Homer, K. Jakubczak, B. Rus, I. J. Kim, C. M. Kim, G. H. Lee, C. H. Nam, V. Hajkova, J. Chalupsky, and L. Juha, J. Appl. Phys. 105, 026105 (2009).

${ }^{14}$ J. J. Rocca, V. Shlyaptsev, F. G. Tomasel, O. D. Cortazar, D. Hartshorn, and J. L. A. Chilla, Phys. Rev. Lett. 73, 2192 (1994).

${ }^{15}$ T. Y. Choi, D. J. Hwang, and C. P. Grigoropoulos, Appl. Surf. Sci. 197-198, 720 (2002).

${ }^{16}$ I. H. Chowdhury, X. Xu, and A. M. Weiner, Appl. Phys. A 83, 49 (2006).

${ }^{17}$ M. Li, S. Menon, J. P. Nibarger, and G. N. Gibson, Phys. Rev. Lett. 82, 2394 (1999).

${ }^{18}$ K. Sugioka, S. Wada, H. Tashiro, K. Toyoda, Y. Ohnuma, and A. Nakamura, Appl. Phys. Lett. 67, 2789 (1995).

${ }^{19}$ K. Obata, K. Sugioka, T. Akane, N. Aoki, K. Toyoda, and K. Midorikawa, Appl. Phys. A 73, 755 (2001).

${ }^{20}$ B. C. Stuart, M. D. Feit, S. Herman, A. M. Rubenchik, B. W. Shore, and M. D. Perry, Phys. Rev. B 53, 1749 (1996).

${ }^{21}$ M. Lenzner, J. Kruger, S. Sartania, Z. Cheng, C. Spielmann, G. Mourou, W. Kautek, and F. Krausz, Phys. Rev. Lett. 80, 4076 (1998).

${ }^{22}$ L. Jiang and H. Tsai, Int. J. Heat Mass Transfer 48, 487 (2005). 